\title{
Comparative genomic analysis of Lactobacillus plantarum ZJ316 reveals its genetic adaptation and potential probiotic profiles ${ }^{* \#}$
}

\author{
Ping $\mathrm{LI}^{1}$, Xuan $\mathrm{LI}^{1,2}$, Qing GU ${ }^{\dagger * 1}$, Xiu-yu LOU ${ }^{1}$, Xiao-mei ZHANG ${ }^{1}$, Da-feng SONG ${ }^{1}$, Chen ZHANG ${ }^{1}$ \\ ( ${ }^{1}$ Key Laboratory for Food Microbial Technology of Zhejiang Province, College of Food Science and Biotechnology, \\ Zhejiang Gongshang University, Hangzhou 310018, China) \\ $\left({ }^{2}\right.$ College of Food and Bioengineering, Henan University of Science and Technology, Luoyang 471023, China) \\ †E-mail: guqing2002@hotmail.com \\ Received Apr. 20, 2016; Revision accepted May 24, 2016; Crosschecked July 13, 2016
}

\begin{abstract}
Objective: In previous studies, Lactobacillus plantarum ZJ316 showed probiotic properties, such as antimicrobial activity against various pathogens and the capacity to significantly improve pig growth and pork quality. The purpose of this study was to reveal the genes potentially related to its genetic adaptation and probiotic profiles based on comparative genomic analysis. Methods: The genome sequence of $L$. plantarum ZJ316 was compared with those of eight $L$. plantarum strains deposited in GenBank. BLASTN, Mauve, and MUMmer programs were used for genome alignment and comparison. CRISPRFinder was applied for searching the clustered regularly interspaced short palindromic repeats (CRISPRs). Results: We identified genes that encode proteins related to genetic adaptation and probiotic profiles, including carbohydrate transport and metabolism, proteolytic enzyme systems and amino acid biosynthesis, CRISPR adaptive immunity, stress responses, bile salt resistance, ability to adhere to the host intestinal wall, exopolysaccharide (EPS) biosynthesis, and bacteriocin biosynthesis. Conclusions: Comparative characterization of the $L$. plantarum ZJ316 genome provided the genetic basis for further elucidating the functional mechanisms of its probiotic properties. ZJ316 could be considered a potential probiotic candidate.
\end{abstract}

Key words: Lactobacillus plantarum ZJ316, Comparative genomics, Probiotics, Adaptation http://dx.doi.org/10.1631/jzus.B1600176

CLC number: Q93

\section{Introduction}

Lactic acid bacteria (LAB) species, including lactobacilli and bifidobacteria, are relatively abundant

\footnotetext{
${ }^{\ddagger}$ Corresponding author

* Project supported by the International Science \& Technology Cooperation Program of China (No. 2013DFA32330), the National Natural Science Foundation of China (Nos. 31540044 and 31271821), the National High-Tech R \& D Program (863) of China (No. 2014AA022210-08), and the Natural Science Foundation of Zhejiang Province (No. LY16C200002), China

\# Electronic supplementary materials: The online version of this article (http://dx.doi.org/10.1631/jzus.B1600176) contains supplementary materials, which are available to authorized users

(1D) ORCID: Qing GU, http://orcid.org/0000-0001-5394-6615

(C) Zhejiang University and Springer-Verlag Berlin Heidelberg 2016
}

inhabitants of the gastrointestinal tract (GIT) of humans and animals. These bacteria are generally regarded as safe (GRAS) and are currently used as probiotics (Presti et al., 2015). The FAO/WHO (Food and Agriculture Organization of the United Nations/ World Health Organization) has defined a probiotic as "live microorganisms which when administered in adequate amounts confer a health benefit on the host" (Hill et al., 2014). In general, probiotics should be capable of dealing with stressful conditions (including in vitro environmental stresses and in vivo human GIT conditions such as acidic $\mathrm{pH}$ and bile salts), and have antimicrobial activity against potential pathogens, the ability to reduce pathogen adhesion and adhere to human epithelial cells. 
Many LAB species, in conjunction with other members of the intestinal microbiota, are believed to contribute to host nutrition, intestinal $\mathrm{pH}$, cell proliferation and differentiation, the immune system, and its innate and acquired responses to pathogens. These perceived health benefits of $\mathrm{LAB}$, which are attributed to the production of antimicrobial metabolites such as bacteriocin, inhibitory enzymes, and organic acids, have driven the commercial exploitation of LAB species as active components of many functional foods and therapeutic adjuncts (Fernandez et al., 2003; Citar et al., 2015).

Lactobacillus plantarum is one member of the $\mathrm{LAB}$ probiotic species that can be isolated from a large variety of environmental niches, including human saliva, grass silage, kimchi, pickled cabbage, and cheese (Siezen and van Hylckama Vlieg, 2011). Some strains, such as ST-III (Wang et al., 2011), have been used as starter cultures or probiotics in the food industry. L. plantarum ZJ316 was originally isolated from fecal samples of healthy infants and showed many probiotic properties such as significantly improving pig growth and pork quality, and antimicrobial activity against various pathogens in vitro including Staphylococcus aureus, Escherichia coli, Salmonella enterica, and Listeria monocytogenes (Suo et al., 2012). However, knowledge of the molecular mechanisms responsible for its probiotic properties is still limited.

Comparative genomic analysis from multiple species or strains can provide insights into the functional and evolutionary processes of genomes. In the present study, we aimed to reveal the genes that might be related to genetic adaptation and probiotic profiles of ZJ316 based on comparative genomic analysis. The whole genome sequences of ZJ316, which have been sequenced by our lab (Li et al., 2013), and other L. plantarum strains such as WCFS1 (Siezen et al., 2012), JDM1 (Zhang et al., 2009), ST-III (Wang et al., 2011) and NC8 (Axelsson et al., 2012) allowed us to annotate the genome of $\mathrm{ZJ} 316$ and make further investigations. Analysis of the predicted genes, together with comparisons to the genomes of other L. plantarum strains, revealed that this bacterium has undergone specific genetic adaptations to colonize and survive in the intestinal tract, and encodes various probiotic related genes.

\section{Materials and methods}

\subsection{Genome sequences of $L$. plantarum strains}

Whole genome sequences of $L$. plantarum ZJ316 have been reported by our lab previously ( $\mathrm{Li}$ et al., 2013), and are deposited in GenBank under accession number CP004082. The sequences and annotations of another eight $L$. plantarum strains studied here were obtained from the NCBI (http://www.ncbi.nlm.nih.gov): L. plantarum WCFS1 (NC_004567), L. plantarum JDM1 (NC_012984), L. plantarum subsp. plantarum ST-III (NC_014554), $L$. plantarum subsp. plantarum P-8 (NC_021224), L. plantarum 16 (NC_021514), L. plantarum subsp. plantarum ATCC 14917 (NZ_ACGZ00000000), L. plantarum subsp. plantarum NC8 (AGRI00000000), and L. plantarum UCMA 3037 (APHP00000000).

\subsection{Bioinformatic analyses}

Whole genome comparisons of the Lactobacillus strains were performed using BLASTN and Mauve alignments (Siezen et al., 2010). Genome synteny comparisons were performed using the set of genomes mentioned above. Whole genomes were compared at the nucleotide level using the MUMmer programs with default parameters (Kurtz et al., 2004) and were viewed using the Artemis comparison tool (ACT) (Carver et al., 2008). Clustered regularly interspaced short palindromic repeats (CRISPRs) were analyzed using CRISPRFinder (Grissa et al., 2007).

\subsection{Phylogenetic tree construction}

A phylogenetic tree was constructed based on genome context networks as described by Ding et al. (2008). Briefly, we constructed the gene context networks from 19 Lactobacillus strains and determined the phylogeny based on the pair-wise similarity of these 19 genomes. The phylogenetic tree was constructed using TreeView.

\section{Results and discussion}

\subsection{General genomic characteristics of $L$. planta- rum ZJ316}

A phylogenetic tree shows the phylogenetic relationships between $\mathrm{ZJ} 316$ and other strains from the 
genus Lactobacillus (Fig. 1). This organism formed a distinct branch with $L$. plantarum strains, and $L$. plantarum IPLA88 was found to be the closest evolutionary relative of strain ZJ316. A total of 3159 genes were predicted as being protein coding sequences (CDSs, about $85 \%$ of the genome), with an average length of $858 \mathrm{bp}$. The remaining intergenic regions had an average length of $144 \mathrm{bp}$. Note that this average length of intergenic regions is shorter than that of other known L. plantarum genomes (164 bp), indicating that L. plantarum ZJ316 has a more compact genome. Among the predicted CDSs, 2563 proteins of $L$. plantarum ZJ316 were functionally categorized, and the proportions in each category were compared with those of other L. plantarum genomes (Table 1 and Fig. 2) (Tatusov et al., 2000). Among all the L. plantarum strains, L. plantarum ZJ316 has the most genes that function in defense mechanisms [V], cell wall/membrane/envelope biogenesis, outer membrane [M], amino acid transport and metabolism [E], lipid transport and metabolism [I], inorganic ion transport and metabolism [P], and secondary metabolite biosynthesis, transport and catabolism [Q]. On the other hand, it has the fewest genes involved in signal transduction mechanisms [T]. Such an extensive genetic adaptation to material transport and metabolism is shared, to a high degree, with enteric $L$. plantarum and likely represents a specific genetic adaptation of bacteria residing in the GIT.
L. plantarum ZJ316 has three plasmids, pLP-ZJ101, pLP-ZJ102, and pLP-ZJ103, encoding 117 genes. A number of plasmids from LAB have been shown to encode multiple important phenotypic traits. Similarly, pLP-ZJ101 is predicted possibly to encode calcium-transporting ATPase (pZJ101_03), relaxase (pZJ101_07), and integrase (pZJ101_15). Plasmid pLP-ZJ102 encodes oligo-1, 6-glucosidase (pZJ102_27), and polysaccharide biosynthesis proteins (pZJ102_48, pZJ102_49, and pZJ102_50). Plasmid pLP-ZJ103 encodes the potassium transport system protein Kup1 (pZJ103_07), the manganese transport protein $\mathrm{MntH}$ (pZJ103_22), as well as the glycine/betaine/carnitine ABC transporters ProX, ProW, and ProV (pZJ103_31-pZJ103_33), which were also found previously in pST-III (Chen et al., 2012). These proteins are thought to be involved in a multi-component binding-protein-dependent transport system for glycine, betaine, and carnitine, allowing accumulation of these compounds to high levels inside the cell in response to increased external osmolarity, which is related to adaptation to the GIT environment.

\subsection{Central carbon metabolism and lifestyle adaptations}

L. plantarum is a versatile and flexible organism and is able to grow on a wide variety of sugar sources. This phenotypic trait is reflected by the high

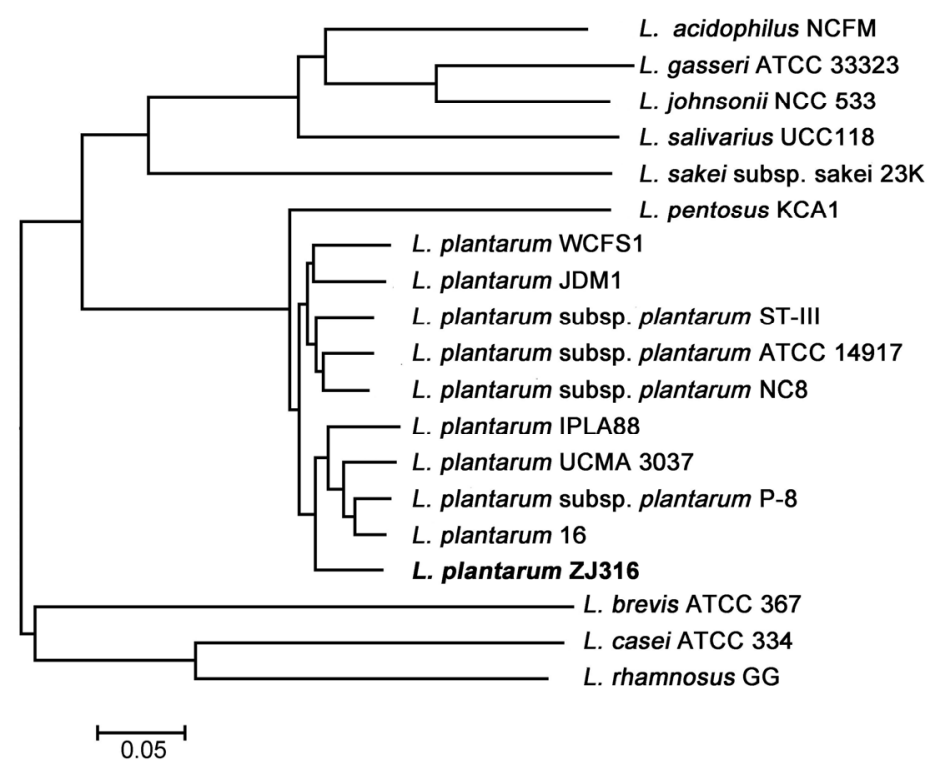

Fig. 1 Phylogenetic tree of ZJ316 and other Lactobacillus strains

Gene context networks were constructed from 19 Lactobacillus strains, and the homologs of all the strains were identified through gapped BLASTP 
Table 1 Clusters of orthologous group (COG) functional categories in 10 completely sequenced genomes of L. plantarum strains

\begin{tabular}{|c|c|c|c|c|c|c|c|c|c|c|}
\hline \multirow{2}{*}{$\begin{array}{l}\mathrm{COG} \\
\text { code }\end{array}$} & \multicolumn{10}{|c|}{ Strain } \\
\hline & ZJ316 & WCFS1 & ST-III & JDM1 & P-8 & 16 & $\begin{array}{l}\text { ATCC } \\
14917\end{array}$ & $\mathrm{NC} 8$ & $\begin{array}{c}\text { UCMA } \\
3037\end{array}$ & IPLA88 \\
\hline$[\mathrm{J}]$ & 150 & 155 & 152 & 152 & 152 & 149 & 153 & 153 & 153 & 154 \\
\hline$[\mathrm{A}]$ & 0 & 0 & 0 & 0 & 0 & 0 & 0 & 0 & 0 & 0 \\
\hline$[\mathrm{K}]$ & 290 & 305 & 301 & 294 & 265 & 270 & 292 & 292 & 275 & 284 \\
\hline [L] & 170 & 157 & 137 & 136 & 190 & 158 & 121 & 121 & 168 & 177 \\
\hline [B] & 0 & 0 & 0 & 0 & 0 & 0 & 0 & 0 & 0 & 0 \\
\hline [D] & 27 & 26 & 26 & 24 & 24 & 26 & 26 & 26 & 27 & 28 \\
\hline$[\mathrm{Y}]$ & 0 & 0 & 0 & 0 & 0 & 0 & 0 & 0 & 0 & 0 \\
\hline$[\mathrm{V}]$ & 63 & 60 & 60 & 60 & 60 & 62 & 56 & 56 & 61 & 59 \\
\hline$[\mathrm{T}]$ & 88 & 100 & 95 & 100 & 89 & 93 & 94 & 94 & 95 & 99 \\
\hline$[\mathrm{M}]$ & 160 & 156 & 153 & 142 & 129 & 130 & 152 & 152 & 140 & 146 \\
\hline$[\mathrm{N}]$ & 7 & 11 & 7 & 9 & 7 & 8 & 8 & 8 & 6 & 6 \\
\hline$[\mathrm{Z}]$ & 0 & 0 & 0 & 0 & 0 & 0 & 0 & 0 & 0 & 0 \\
\hline [W] & 0 & 0 & 0 & 0 & 0 & 0 & 0 & 0 & 0 & 0 \\
\hline$[\mathrm{U}]$ & 23 & 26 & 24 & 25 & 23 & 21 & 25 & 25 & 21 & 25 \\
\hline$[\mathrm{O}]$ & 62 & 62 & 62 & 64 & 61 & 61 & 61 & 61 & 61 & 62 \\
\hline$[\mathrm{C}]$ & 112 & 114 & 107 & 112 & 103 & 106 & 106 & 106 & 107 & 106 \\
\hline [G] & 278 & 313 & 289 & 305 & 242 & 248 & 284 & 284 & 247 & 248 \\
\hline$[\mathrm{E}]$ & 267 & 267 & 250 & 259 & 243 & 247 & 251 & 251 & 252 & 251 \\
\hline$[\mathrm{F}]$ & 85 & 87 & 81 & 85 & 84 & 85 & 86 & 86 & 92 & 85 \\
\hline$[\mathrm{H}]$ & 78 & 91 & 77 & 93 & 77 & 77 & 78 & 78 & 80 & 76 \\
\hline$[\mathrm{I}]$ & 77 & 75 & 73 & 72 & 69 & 71 & 70 & 70 & 70 & 72 \\
\hline$[\mathrm{P}]$ & 171 & 169 & 157 & 167 & 148 & 159 & 163 & 163 & 152 & 164 \\
\hline [Q] & 42 & 42 & 38 & 38 & 37 & 36 & 40 & 40 & 37 & 38 \\
\hline [R] & 413 & 421 & 394 & 404 & 369 & 382 & 405 & 405 & 381 & 390 \\
\hline$[\mathrm{S}]$ & 220 & 224 & 220 & 223 & 212 & 209 & 221 & 221 & 215 & 222 \\
\hline $\begin{array}{l}\text { No related } \\
\text { COG }\end{array}$ & 180 & 175 & 168 & 159 & 157 & 152 & 174 & 174 & 153 & 203 \\
\hline No hits & 596 & 437 & 499 & 419 & 497 & 338 & 386 & 386 & 484 & 573 \\
\hline Total & 3159 & 3058 & 2996 & 2948 & 2893 & 2778 & 2868 & 2868 & 2932 & 3116 \\
\hline
\end{tabular}

$[\mathrm{J}]$ translation, $[\mathrm{A}]$ RNA processing and modification, $[\mathrm{K}]$ transcription, $[\mathrm{L}]$ replication, recombination and repair, $[\mathrm{B}]$ chromatin structure and dynamics, $[\mathrm{D}]$ cell cycle control, mitosis and meiosis, $[\mathrm{Y}]$ nuclear structure, $[\mathrm{V}]$ defense mechanisms, $[\mathrm{T}]$ signal transduction mechanisms, $[\mathrm{M}]$ cell wall/membrane biogenesis, $[\mathrm{N}]$ cell motility, $[\mathrm{Z}]$ cytoskeleton, $[\mathrm{W}]$ extracellular structures, $[\mathrm{U}]$ intracellular trafficking and secretion, $[\mathrm{O}]$ posttranslational modification, protein turnover, chaperones, $[\mathrm{C}]$ energy production and conversion, $[\mathrm{G}]$ carbohydrate transport and metabolism, $[\mathrm{E}]$ amino acid transport and metabolism, $[\mathrm{F}]$ nucleotide transport and metabolism, $[\mathrm{H}]$ coenzyme transport and metabolism, [I] lipid transport and metabolism, [P] inorganic ion transport and metabolism, [Q] secondary metabolites biosynthesis, transport and catabolism, $[\mathrm{R}]$ general function prediction only, $[\mathrm{S}]$ function unknown

number of genes encoding putative sugar transporters. The majority of these transporters are predicted phosphoenolpyruvate (PEP)-dependent sugar phosphotransferase systems (PTSs). L. plantarum ZJ316 encodes 17 complete PTS enzyme II complexes and several incomplete complexes (Table S1). Once internalized, sugars are used as carbon sources for growth and for the generation of energy through fermentation. L. plantarum is usually grouped with the facultative heterofermentative lactobacilli, and sugars can be fermented via the Embden-MeyerhofParnas (EMP) pathway or the phosphoketolase pathway, leading to homolactic or heterolactic fermentation profiles, respectively (Kandler, 1983). In support of this classification, genes encoding enzymes involved in the EMP pathway were found in ZJ316. 


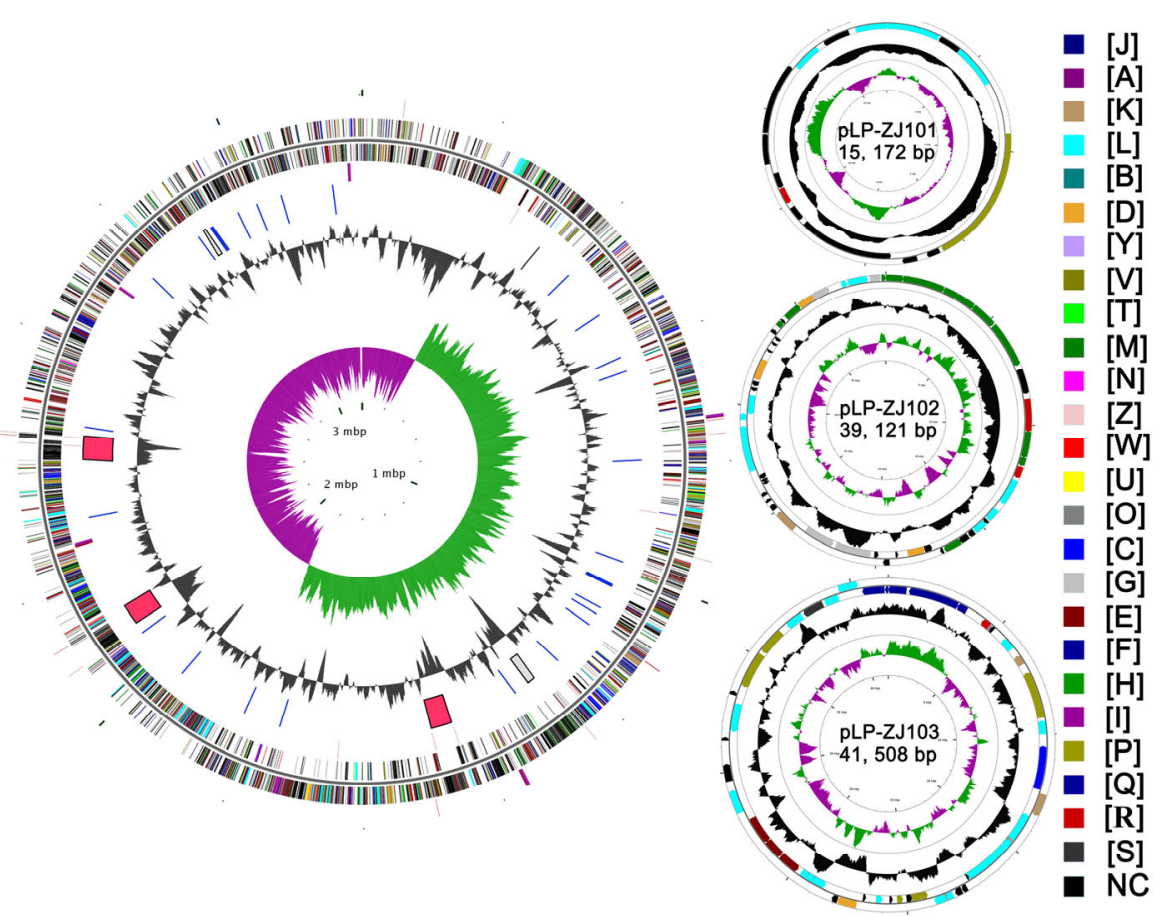

Fig. 2 Circular genome map of $L$. plantarum ZJ316

From the innermost circles, circle (1) illustrates the $\mathrm{GC}$ skew $((\mathrm{G}-\mathrm{C}) /(\mathrm{G}+\mathrm{C}))$, where values $>0$ are in green, and values $<0$ are in purple. Circle (2) highlights the $(\mathrm{G}+\mathrm{C}) \%$ deviation from the mean $(44.65 \%)$. Circle (3) denotes intact prophages in red, incomplete prophages in grey, and CRISPR repeats in black. Circle (4) indicates rRNAs (depicted in purple) and tRNAs (depicted in red) in the lagging replication strand. Coding regions are indicated by strand, and the color corresponds to the COG functional assignments; the lagging replication strand is shown in circle (5) and the leading replication strand in circle (6). Circle (7) denotes rRNA and tRNA genes in the leading replication strand (Note: for interpretation of the references to color in this figure legend, the reader is referred to the web version of this article)

However, ZJ316 and ST-III lack both xylulose-5-P phosphoketolase and deoxyribose-phosphate aldolase, which prevent the conversion of D-xylulose 5-phosphate to D-glyceraldehyde 3-phosphate and the conversion between 2-deoxy-D-ribose 5-phosphate and D-glyceraldehyde 3-phosphate. As expected, the L. plantarum chromosome does not encode an intact citrate acid cycle. Moreover, the ara, rha and iol gene clusters responsible for arabinose, rhamnose, and myo-inositol utilization are not present in ZJ316, which indicates that the range of carbohydrate metabolism is constrained.

In particular, the $129 \mathrm{~kb}$ region from 92000 to 221000 almost exclusively encodes proteins for sugar transport, metabolism, and regulation. This region is much shorter than the $213 \mathrm{~kb}$ region in the other L. plantarum strains (Fig. 3). Moreover, this entire region has a lower $\mathrm{G}+\mathrm{C}$ content $(41.41 \%)$ than the rest of the genome (Fig. 2), suggesting that many genes may have been acquired through horizontal gene transfer (HGT). This finding supports the hypothesis that this part of the L. plantarum chromosome represents a lifestyle-adaptation region that is used to adapt effectively to changes in conditions encountered in certain environmental niches in which this microbe is found.

\subsection{Proteolytic enzyme systems and amino acid biosynthesis}

LAB generally inhabit protein-rich environments and are equipped with protein-degradation machinery to create a selective advantage for growth under these conditions. All L. plantarum genomes appear to encode the primary enzyme membraneassociated lipoprotein PrtM for the primary breakdown of proteins and large polypeptide utilization (Haandrikman et al., 1991). The presence of prtM genes in both ZJ316 (zj316_1494 and zj316_3030) and other $L$. plantarum strains suggests that the organisms can digest large proteins extracellularly and 


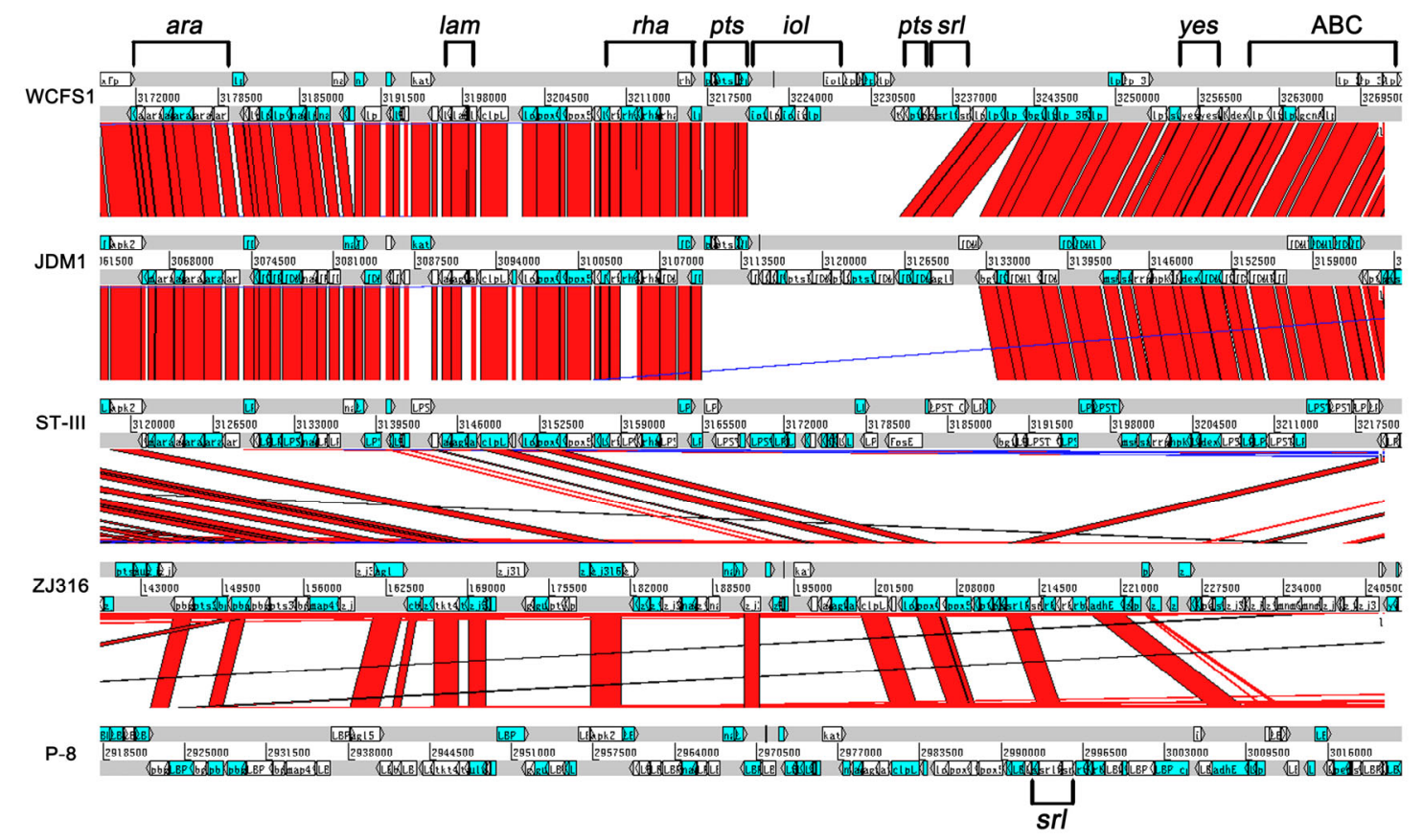

Fig. 3 Diversity of lifestyle adaptation region genes in L. plantarum strains

Comparison of genome organization surrounding the large cluster of lifestyle adaptation region genes. Genes are represented by blue and white arrows in the forward and reverse strands. The red connecting bars indicate high sequence identity and the blue bars a reverse orientation. The consecutive gene clusters, including arabinose biosynthesis (ara), auto-inducing peptide (lam), rhamnose biosynthesis (rha), PTS system (pts), myo-inositol biosynthesis (iol), sorbitol operon ( $r r l)$, two-component (yes), and $\mathrm{ABC}$ transporter $(\mathrm{ABC})$, are indicated in WCFS1 (Note: for interpretation of the references to color in this figure legend, the reader is referred to the web version of this article)

generate primary protein-degradation products that are internalized by uptake systems (Opp and Dtp clusters). Two separated Opp clusters (zj316_1286zj316_1290 and zj316_3100-zj316_3104), which are di- and oligo-peptide transporting systems, were predicted. This prediction is in contrast to the single cluster found in the other L. plantarum strains. As in other L. plantarum strains, only one permease-type transporter for di- and tri-peptides ( $\left.d t p \mathrm{~T}, \mathrm{zj} 316 \_0697\right)$ was located in ZJ316. Scattered throughout the genome, two additional genes coding for distinct periplasmatic components (OppA) were identified and likely broaden the Opp-transporter specificities (zj316_0304 and zj316_0801).

Once internalized, these peptides are degraded by a variety of peptidases and proteases, which have been extensively studied in LAB (Savijoki et al., 2006). ZJ316 has 34 genes encoding intracellular peptidases and 23 genes encoding proteases of different specificity (Table S2). Like other L. plantarum strains, ZJ316 encodes the complete biosynthetic pathways of most amino acids except valine, leucine, and isoleucine.

\subsection{Stress response}

Despite the presence of stress response machinery in all eight $L$. plantarum strains, special proteins such as the ImpB/MucB/SamB family proteins and proteins of nitrate/sulfonate/bicarbonate $\mathrm{ABC}$ transporter (zj316_0670 and zj316_0671) were identified in ZJ316. The nitrate/sulfonate/bicarbonate $\mathrm{ABC}$ transporter, which also can be found in strain ST-III, is up-regulated in response to salt-stress (Huang et al., 2006). It may increase the efficiency of inorganic salt uptake and give the bacteria a way to survive and compete in the gut niche (Lurie-Weinberger et al., 2012). Two genes, zj316_2874 and zj316_2875, code the $\mathrm{ImpB} / \mathrm{MucB} / \mathrm{SamB}$ family proteins, which are involved in ultraviolet (UV) protection. zj316_0075 and zj316_2984 encode two DNA-binding ferritinlike proteins (Dps family proteins). Both the ImpB/ MucB/SamB and Dps family proteins play central roles in protecting DNA from oxidative damage by directly binding to DNA (Zhao et al., 2002; Chang et al., 2006). 


\subsection{Bile salt hydrolase}

Bile is one of the most serious obstacles to bacterial survival in a mammalian gut. Conjugated bile acids (CBAs) have been reported to influence the intestinal microflora through direct antimicrobial effects, upregulation of host mucosal defenses, or synergistic action of both (Begley et al., 2006; Kumar et al., 2012). To survive in a gut, lactobacilli produce bile salt hydrolases (BSH) to deconjugate the amino acid moiety from CBAs. BSH activity has been detected and bsh genes identified in Lactobacillus, especially in the strains associated with the intestinal tract. Interestingly, two bsh genes (zj316_0150 and zj316_0281) were detected in the feces-derived strain ZJ316. The feature of multiple BSH homologs is also found in WCFS1, which has four bsh genes (Bron et al., 2006). Begley et al. (2006) speculated that the multiple BSH feature may help the bacteria to tolerate different types of bile or perhaps promote bile adaptation, thus ensuring maximal survival of the bacteria under changing environmental conditions. ZJ316 might show good bile tolerance based on these genomic features.

\subsection{Adhesion ability}

Adhesion of bacteria to mucosal surfaces is necessary both to colonize the small intestinal tract and deliver the health benefits; thus adhesion ability is an important selection criterion for probiotics (Lavilla-Lerma et al., 2013). Adhesion capacity might be associated with certain surface proteins, fatty acids, and exopolysaccharides (EPS). EF-Tu is a novel identified surface protein that has the characteristics of an adhesion factor and is capable of inducing a proinflammatory response. It functions as an adhesion-like factor in Bifidobacterium (Yuan et al., 2008). EF-Tu was encoded by gene zj316_2121 in ZJ316, suggesting potential adhesion of the strain in the GIT.

\subsection{Capsular polysaccharide (CPS) biosynthesis genes}

EPS produced by LAB have received increasing attention because of their capacity to improve the textural properties of fermented dairy and non-dairy products (Yang et al., 2010). CPS in LAB is the EPS attached at the bacterial surface and has been shown to be important for the colonization, adhesion, stress resistance, host-bacteria interactions, as well as immunomodulation of Lactobacillus strains. Recent evidence also shows that the cps clusters encoding polysaccharides contribute to the $L$. plantarum WCFS1 cell surface architecture and are probably related to TLR2 signaling (Remus et al., 2012). Three $c p s$ clusters (cps3, cps 4 , and parts of the $c p s 2$ clusters) were identified in ZJ316, encoding proteins involved in biosynthesis and the export of CPSs (Fig. 4). Strain ST-III shares most of the cps clusters with ZJ316. However, strains JDM1, UCMA 3037, P8, and 16 have only the $c p s 4$ cluster. Strain WCFS1 has four $c p s$ clusters (cps 1, cps 2, cps3, and cps4) and the largest number of cps genes (Remus et al., 2012). It has been reported that many probiotic properties of ST-III, including cholesterol removal, may relate to CPSs (Wang et al., 2011). We speculate that the presence of cps clusters in ZJ316 might be of benefit for its probiotic properties and gut adaptation.

\subsection{Plantaricin biosynthesis genes}

Bacteriocins are antimicrobial peptides produced by bacteria that inhibit the growth of similar or closely related bacterial strains, and have been considered as potential drug candidates for replacing antibiotics (Alvarez-Sieiro et al., 2016). Bacteriocins produced by $L$. plantarum strains facilitate their competitive ability in the environment and maximize their survival chances. The plantaricin ( $p l n)$ locus is responsible for bacteriocin biosynthesis in $L$. plantarum. The pln gene cluster of $L$. plantarum contains about 25 genes (Fig. 5) and encodes various class II bacteriocins (Diep et al., 2009; Nissen-Meyer et al., 2010). It was found to be a highly variable and mosaic region, with some parts relatively conserved and other parts less conserved (Diep et al., 2009; Saenz et al., 2009). The pln loci of nine fully sequenced $L$. plantarum strains were also highly variable (Fig. 5). Twenty-two genes related to class II bacteriocins were identified in the genome of ZJ316. ZJ316 has genes encoding the two-peptide plantaricin PlnJK and the inducible class IIb plantaricin $\mathrm{NC} 8 \beta \alpha$, and the corresponding immunity protein NC8c (Maldonado et al., 2003). ZJ316 and NC8 share the regulatory operon, which includes the inducing peptide PLNC8IF and the histidine protein kinase PLNC8K (Maldonado et al., 2004), while the other strains use a different regulatory operon, $p \ln \mathrm{ABCD}$. 


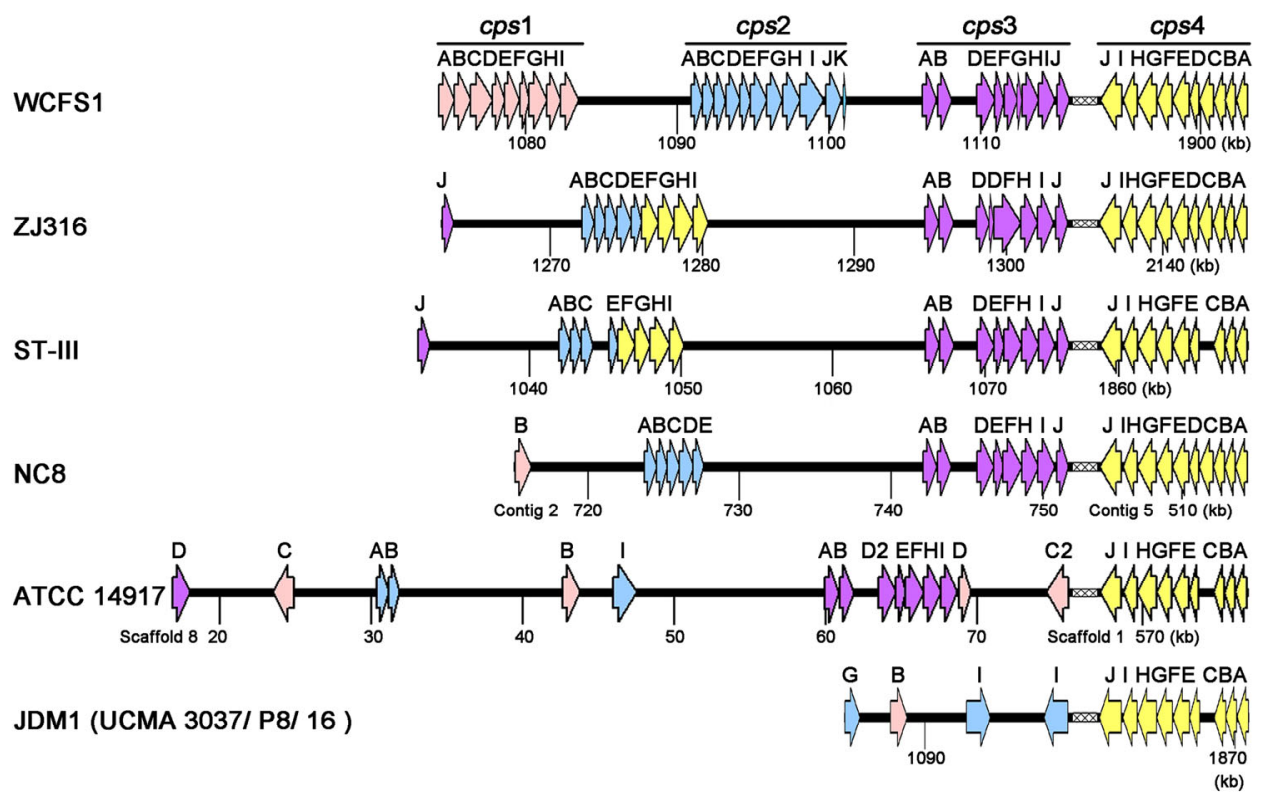

Fig. 4 Diversity of capsular polysaccharide biosynthesis genes in the completely sequenced genomes of $L$. plantarum The four $c p s$ gene clusters are represented by pink, blue, purple, and yellow arrows, respectively (Note: for interpretation of the references to color in this figure legend, the reader is referred to the web version of this article)

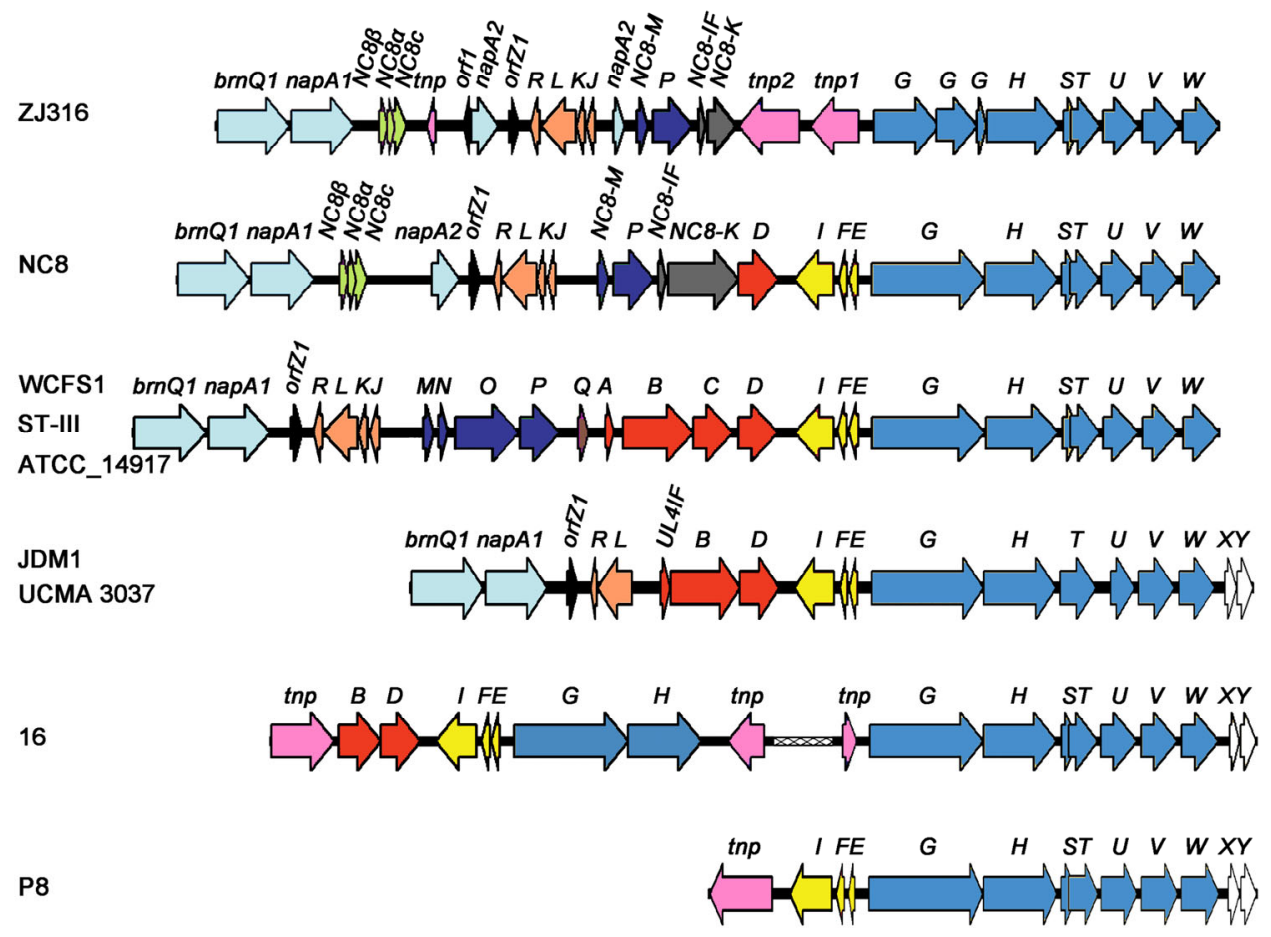

Fig. 5 Genetic map of the pln loci of different L. plantarum strains

The $p \ln$ genes are represented by arrows with different colors corresponding to each operon. The arrows indicate that the genes are partial sequences according to those published in the GenBank database. Color codes for $p l n$ operons: red/grey for the genes of the regulatory operon; yellow for the $p \ln \mathrm{EFI}$ operon; blue for the $\ln \mathrm{MNOP}$ operon; orange for the $p \ln \mathrm{JKLR}$ operon; pale green for the $p l \mathrm{NC} 8 \beta \alpha \mathrm{c}$ operon; gray-blue for the $\mathrm{ABC}$ transporter system genes; and purple for genes of the transposases. Strain-specific genes are named as open reading frames (ORFs) and are colored in white and black (Note: for interpretation of the references to color in this figure legend, the reader is referred to the web version of this article) 
However, there are no $p \ln \mathrm{C}$ or $p \ln \mathrm{D}$ response regulator orthologs or two-peptide bacteriocin $p \ln \mathrm{EFI}$ operons in ZJ316. The ZJ316 strain could produce at least two class IIb bacteriocins, PlnJK and PlnNC $8 \beta \alpha$, and a class IIc bacteriocin, PlnA, suggesting that this L. plantarum strain is a great producer of bactericins, which could explain its antimicrobial activity against pathogens.

\subsection{CRISPR repeats}

CRISPRs represent a family of DNA repeats that are typically composed of short and highly conserved repeats, interspaced by variable sequences called spacers and are often found adjacent to cas (CRISPRassociated) genes (Sorek et al., 2008). CRISPRs represent the most widely distributed prokaryotic family of repeats and act as defense systems against invasion of foreign genetic material, in particular, phages (Barrangou et al., 2007). L. plantarum ZJ316 contains one CRISPR locus, which belongs to the well-conserved Lsal1 family (Horvath et al., 2009). This locus contains 7 perfect repeats of a 36-bp sequence (5'-GTCTTGAATAGTAGTCATATCAAAC AGGTTTAGAAC-3'). Four cas genes (zj316_0669zj316_0672) are found upstream of the DNA repeats with an average $\mathrm{G}+\mathrm{C}$ content of $42.2 \%$ (vs. $44.6 \%$ for the chromosome), suggesting that either these genes were acquired from an organism with a similar $\mathrm{G}+\mathrm{C}$ content or were acquired in the distant past. The presence of CRISPR loci may increase the genome stability of strain ZJ316, and therefore its adaptation in the environment.

\section{Conclusions}

ZJ316 is a L. plantarum strain isolated from infant feces and has several great probiotic properties. In the present study, we revealed the genes that may be related to its genetic adaptation and probiotic profiles based on comparative genomic analysis. Genes related to carbohydrate transport and metabolism, proteolytic enzyme systems and amino acid biosynthesis, prophages and CRISPR adaptive immunity, stress responses, bile salt resistance, adhesion ability, CPS biosynthesis, and bacteriocin biosynthesis were identified. The feature of bacteriocin biosynthesis may provide clues to explain the antimicrobial activ- ity of ZJ316. Bile salt resistance, adhesion ability, and stress resistances show its potential capability to survive in vitro environmental stresses and in vivo human GIT conditions. This research could provide the genetic basis for further studies elucidating the functional mechanisms of the probiotic properties of ZJ316 and facilitate its consideration as a potential probiotic candidate. Further research, such as transcriptomic and proteomic studies, will allow us to elucidate the physiological importance of the genes identified in bacterial survival, host colonization, pathogen exclusion, and antimicrobial activity.

\section{Compliance with ethics guidelines}

Ping LI, Xuan LI, Qing GU, Xiu-yu LOU, Xiao-mei ZHANG, Da-feng SONG, and Chen ZHANG declare that they have no conclict of interest.

This article does not contain any studies with human or animal subjects performed by any of the authors.

\section{References}

Alvarez-Sieiro, P., Montalban-Lopez, M., Mu, D., et al., 2016. Bacteriocins of lactic acid bacteria: extending the family. Appl. Microbiol. Biotechnol., 100(7):2939-2951. http://dx.doi.org/10.1007/s00253-016-7343-9

Axelsson, L., Rud, I., Naterstad, K., et al., 2012. Genome sequence of the naturally plasmid-free Lactobacillus plantarum strain NC8 (CCUG 61730). J. Bacteriol., 194(9):2391-2392.

http://dx.doi.org/10.1128/JB.00141-12

Barrangou, R., Fremaux, C., Deveau, H., et al., 2007. CRISPR provides acquired resistance against viruses in prokaryotes. Science, 315(5819): 1709-1712. http://dx.doi.org/10.1126/science. 1138140

Begley, M., Hill, C., Gahan, C.G., 2006. Bile salt hydrolase activity in probiotics. Appl. Environ. Microbiol., 72(3): 1729-1738. http://dx.doi.org/10.1128/AEM.72.3.1729-1738.2006

Bron, P.A., Molenaar, D., de Vos, W.M., et al., 2006. DNA micro-array-based identification of bile-responsive genes in Lactobacillus plantarum. J. Appl. Microbiol., 100(4): 728-738. http://dx.doi.org/10.1111/j.1365-2672.2006.02891.x

Carver, T., Berriman, M., Tivey, A., et al., 2008. Artemis and ACT: viewing, annotating and comparing sequences stored in a relational database. Bioinformatics, 24(23):2672-2676. http://dx.doi.org/10.1093/bioinformatics/btn529

Chang, W., Small, D.A., Toghrol, F., et al., 2006. Global transcriptome analysis of Staphylococcus aureus response to hydrogen peroxide. J. Bacteriol., 188(4):1648-1659. http://dx.doi.org/10.1128/JB.188.4.1648-1659.2006

Chen, C., Ai, L., Zhou, F., et al., 2012. Complete nucleotide sequence of plasmid pST-III from Lactobacillus plantarum ST-III. Plasmid, 67(3):236-244. http://dx.doi.org/10.1016/j.plasmid.2011.12.005 
Citar, M., Hacin, B., Tompa, G., et al., 2015. Human intestinal mucosa-associated Lactobacillus and Bifidobacterium strains with probiotic properties modulate IL-10, IL-6 and IL-12 gene expression in THP-1 cells. Benef. Microbes, 6(3):325-336. http://dx.doi.org/10.3920/BM2014.0081

Diep, D.B., Straume, D., Kjos, M., et al., 2009. An overview of the mosaic bacteriocin pln loci from Lactobacillus plantarum. Peptides, 30(8):1562-1574. http://dx.doi.org/10.1016/j.peptides.2009.05.014

Ding, G., Yu, Z., Zhao, J., et al., 2008. Tree of life based on genome context networks. PLOS ONE, 3(10):e3357. http://dx.doi.org/10.1371/journal.pone.0003357

Fernandez, M.F., Boris, S., Barbes, C., 2003. Probiotic properties of human lactobacilli strains to be used in the gastrointestinal tract. J. Appl. Microbiol., 94(3):449-455. http://dx.doi.org/10.1046/j.1365-2672.2003.01850.x

Grissa, I., Vergnaud, G., Pourcel, C., 2007. CRISPRFinder: a web tool to identify clustered regularly interspaced short palindromic repeats. Nucleic Acids Res., 35(Suppl. 2): W52-W57. http://dx.doi.org/10.1093/nar/gkm360

Haandrikman, A.J., Meesters, R., Laan, H., et al., 1991. Processing of the lactococcal extracellular serine proteinase. Appl. Environ. Microbiol., 57(7):1899-1904.

Hill, C., Guarner, F., Reid, G., et al., 2014. Expert consensus document. The international scientific association for probiotics and prebiotics consensus statement on the scope and appropriate use of the term probiotic. Nat. Rev. Gastroenterol. Hepatol., 11(8):506-514. http://dx.doi.org/10.1038/nrgastro.2014.66

Horvath, P., Coute-Monvoisin, A.C., Romero, D.A., et al., 2009. Comparative analysis of CRISPR loci in lactic acid bacteria genomes. Int. J. Food Microbiol., 131(1):62-70. http://dx.doi.org/10.1016/j.ijfoodmicro.2008.05.030

Huang, F., Fulda, S., Hagemann, M., et al., 2006. Proteomic screening of salt-stress-induced changes in plasma membranes of Synechocystis sp. strain PCC 6803. Proteomics, 6(3):910-920. http://dx.doi.org/10.1002/pmic.200500114

Kandler, O., 1983. Carbohydrate metabolism in lactic acid bacteria. Antonie van Leeuwenhoek, 49(3):209-224. http://dx.doi.org/10.1007/BF00399499

Kumar, R., Grover, S., Batish, V.K., 2012. Bile salt hydrolase (Bsh) activity screening of lactobacilli: in vitro selection of indigenous Lactobacillus strains with potential bile salt hydrolysing and cholesterol-lowering ability. Probiotics Antimicrob. Proteins, 4(3):162-172. http://dx.doi.org/10.1007/s12602-012-9101-3

Kurtz, S., Phillippy, A., Delcher, A.L., et al., 2004. Versatile and open software for comparing large genomes. Genome Biol., 5(2):R12. http://dx.doi.org/10.1186/gb-2004-5-2-r12

Lavilla-Lerma, L., Perez-Pulido, R., Martinez-Bueno, M., et al., 2013. Characterization of functional, safety, and gut survival related characteristics of Lactobacillus strains isolated from farmhouse goat's milk cheeses. Int. J. Food Microbiol., 163(2-3):136-145.

http://dx.doi.org/10.1016/j.ijfoodmicro.2013.02.015

Li, X., Gu, Q., Lou, X., et al., 2013. Complete genome sequence of the probiotic Lactobacillus plantarum strain ZJ316. Genome Announc., 1(2):e00094-13. http://dx.doi.org/10.1128/genomeA.00094-13

Lurie-Weinberger, M.N., Peeri, M., Gophna, U., 2012. Contribution of lateral gene transfer to the gene repertoire of a gut-adapted methanogen. Genomics, 99(1):52-58. http://dx.doi.org/10.1016/j.ygeno.2011.10.005

Maldonado, A., Ruiz-Barba, J.L., Jimenez-Diaz, R., 2003. Purification and genetic characterization of plantaricin NC8, a novel coculture-inducible two-peptide bacteriocin from Lactobacillus plantarum NC8. Appl. Environ. Microbiol., 69(1):383-389. http://dx.doi.org/10.1128/AEM.69.1.383-389.2003

Maldonado, A., Ruiz-Barba, J.L., Jimenez-Diaz, R., 2004. Production of plantaricin NC8 by Lactobacillus plantarum NC8 is induced in the presence of different types of Gram-positive bacteria. Arch. Microbiol., 181(1): 8-16. http://dx.doi.org/10.1007/s00203-003-0606-8

Nissen-Meyer, J., Oppegard, C., Rogne, P., et al., 2010. Structure and mode-of-action of the two-peptide (class-IIb) bacteriocins. Probiotics Antimicrob. Proteins, 2(1):52-60. http://dx.doi.org/10.1007/s12602-009-9021-z

Presti, I., D'Orazio, G., Labra, M., et al., 2015. Evaluation of the probiotic properties of new Lactobacillus and Bifidobacterium strains and their in vitro effect. Appl. Microbiol. Biotechnol., 99(13):5613-5626. http://dx.doi.org/10.1007/s00253-015-6482-8

Remus, D.M., van Kranenburg, R., van Swam, I.I., et al., 2012. Impact of 4 Lactobacillus plantarum capsular polysaccharide clusters on surface glycan composition and host cell signaling. Microb. Cell Fact., 11:149. http://dx.doi.org/10.1186/1475-2859-11-149

Saenz, Y., Rojo-Bezares, B., Navarro, L., et al., 2009. Genetic diversity of the pln locus among oenological Lactobacillus plantarum strains. Int. J. Food Microbiol., 134(3): 176-183. http://dx.doi.org/10.1016/j.ijfoodmicro.2009.06.004

Savijoki, K., Ingmer, H., Varmanen, P., 2006. Proteolytic systems of lactic acid bacteria. Appl. Microbiol. Biotechnol., 71(4):394-406. http://dx.doi.org/10.1007/s00253-006-0427-1

Siezen, R.J., van Hylckama Vlieg, J.E., 2011. Genomic diversity and versatility of Lactobacillus plantarum, a natural metabolic engineer. Microb. Cell Fact., 10(Suppl. 1):S3. http://dx.doi.org/10.1186/1475-2859-10-S1-S3

Siezen, R.J., Tzeneva, V.A., Castioni, A., et al., 2010. Phenotypic and genomic diversity of Lactobacillus plantarum strains isolated from various environmental niches. Environ. Microbiol., 12(3):758-773. http://dx.doi.org/10.1111/j.1462-2920.2009.02119.x 
Siezen, R.J., Francke, C., Renckens, B., et al., 2012. Complete resequencing and reannotation of the Lactobacillus plantarum WCFS1 genome. J. Bacteriol., 194(1):195-196. http://dx.doi.org/10.1128/JB.06275-11

Sorek, R., Kunin, V., Hugenholtz, P., 2008. CRISPR-a widespread system that provides acquired resistance against phages in bacteria and archaea. Nat. Rev. Microbiol., 6(3):181-186. http://dx.doi.org/10.1038/nrmicro1793

Suo, C., Yin, Y., Wang, X., et al., 2012. Effects of Lactobacillus plantarum ZJ316 on pig growth and pork quality. BMC Vet. Res., 8:89. http://dx.doi.org/10.1186/1746-6148-8-89

Tatusov, R.L., Galperin, M.Y., Natale, D.A., et al., 2000. The COG database: a tool for genome-scale analysis of protein functions and evolution. Nucleic Acids Res., 28(1):33-36. http://dx.doi.org/10.1093/nar/28.1.33

Wang, Y., Chen, C., Ai, L., et al., 2011. Complete genome sequence of the probiotic Lactobacillus plantarum ST-III. J. Bacteriol., 193(1):313-314. http://dx.doi.org/10.1128/JB.01159-10

Yang, Z., Li, S., Zhang, X., et al., 2010. Capsular and slimepolysaccharide production by Lactobacillus rhamnosus JAAS8 isolated from Chinese sauerkraut: potential application in fermented milk products. J. Biosci. Bioeng., 110(1):53-57. http://dx.doi.org/10.1016/j.jbiosc.2009.12.010

Yuan, J., Wang, B., Sun, Z., et al., 2008. Analysis of hostinducing proteome changes in bifidobacterium longum NCC2705 grown in vivo. J. Proteome Res., 7(1):375-385. http://dx.doi.org/10.1021/pr0704940

Zhang, Z.Y., Liu, C., Zhu, Y.Z., et al., 2009. Complete genome sequence of Lactobacillus plantarum JDM1. J. Bacteriol., 191(15):5020-5021. http://dx.doi.org/10.1128/JB.00587-09

Zhao, G., Ceci, P., Ilari, A., et al., 2002. Iron and hydrogen peroxide detoxification properties of DNA-binding protein from starved cells. A ferritin-like DNA-binding protein of Escherichia coli. J. Biol. Chem., 277(31): 27689-27696.

http://dx.doi.org/10.1074/jbc.M202094200

\section{List of electronic supplementary materials}

Table S1 PTS predicted in L. plantarum ZJ316

Table S2 Peptidases and proteases genes in L. plantarum ZJ316

\section{中文概要}

题 目: 比较基因组学揭示植物乳杆菌 ZJ316 的生境适 应性及潜在益生特性

目 的: 前期研究发现植物乳杆菌 ZJ316 能显著抑制病原 菌, 促进仔猪生长, 提高猪肉质量等, 本研究拟 在 ZJ316 全基因组测序的基础上, 运用比较基因 组学手段揭示与其生境适应性及益生特性相关 基因。

创新点: 首次从基因水平上分析与植物乳杆菌 ZJ316 的生 境适应性、抑菌活性及益生特性等相关的基因, 为进一步揭示其生理功能打下基础。

方 法: 运用 BLASTN、Mauve 和 MUMmer 等将植物乳 杆菌 ZJ316 全基因组序列与已测序的 8 个植物乳 杆菌全基因组序列进行比对及分析; 用 CRISPRFinder 寻找 CRISPR 重复序列。

结 论: 植物乳杆菌 ZJ316 包含碳水化合物的运输和代谢、 蛋白水解酶系统和氨基酸的生物合成等相关基 因，具有 CRISPR、应激反应、耐胆盐、粘附宿 主肠壁、胞外多糖、生物合成和细菌素生物合成 等相关基因。这些基因的功能是其作为益生菌的 重要特征和基础。

关键词: 植物乳杆菌 ZJ316; 比较基因组学; 益生菌; 适 应性 